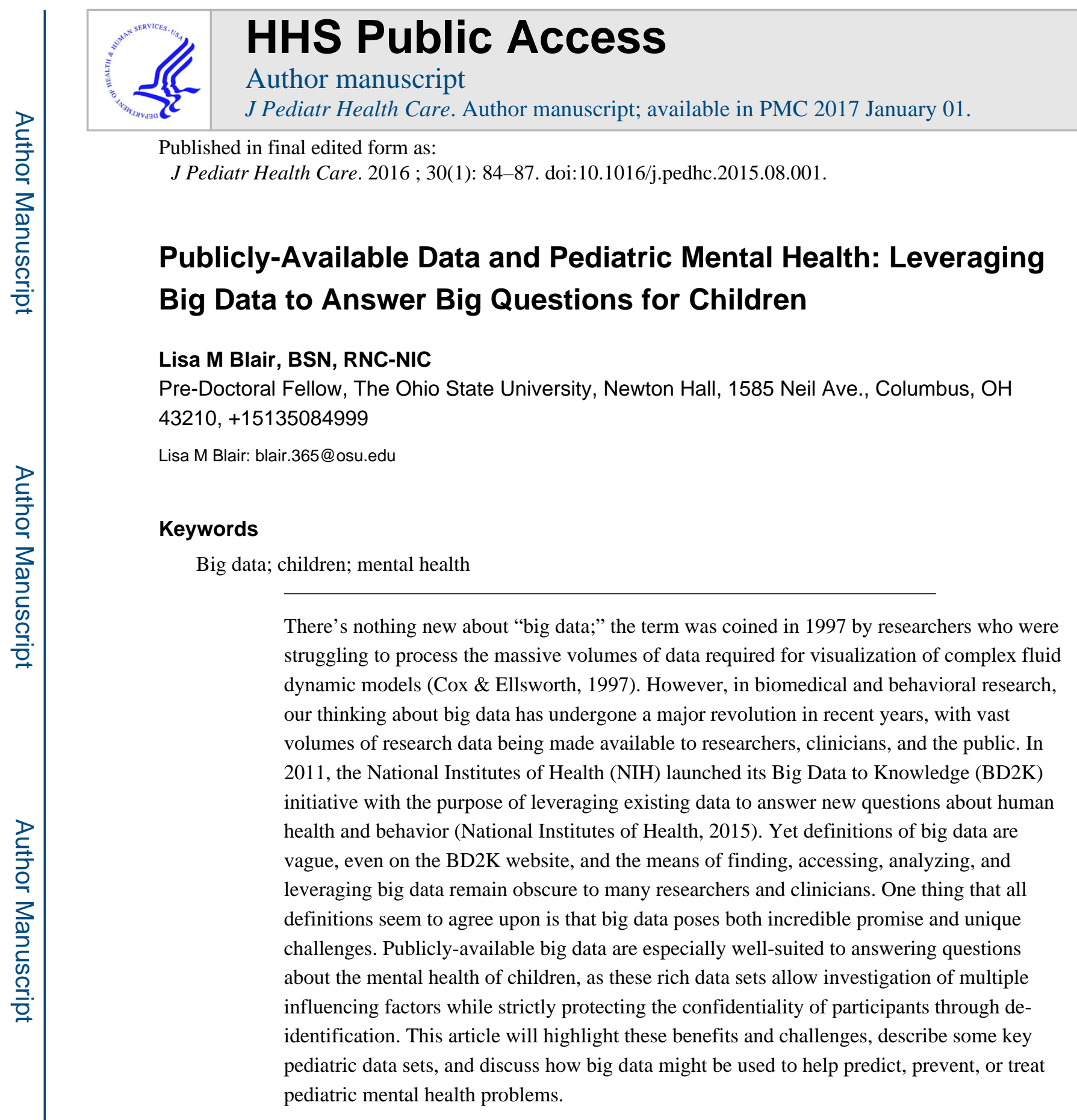

\title{
The Benefits of Publicly-Available Data
}

Publicly-available big data provides a range of substantial benefits to researchers and clinicians with questions about clinical problems. These benefits vary across studies based on design, sample size, and type of data collected, but all publicly-available data share one major benefit: ease of access. Many providers of publicly-available data require only that

Institution of Origination: The Ohio State University

The contents are the responsibility of the authors and do not necessarily represent the official views of the NIH.

Publisher's Disclaimer: This is a PDF file of an unedited manuscript that has been accepted for publication. As a service to our customers we are providing this early version of the manuscript. The manuscript will undergo copyediting, typesetting, and review of the resulting proof before it is published in its final citable form. Please note that during the production process errors may be discovered which could affect the content, and all legal disclaimers that apply to the journal pertain. 
users register with their website in order to obtain the data, while others allow direct download without registration, making the data available not just to researchers, but also to the public.

Timeliness is another major benefit, particularly for longitudinal data that an individual researcher might spend years or decades collecting. With publicly-available data, the time frame from hypothesis to evidence is shortened because the data are already collected, cleaned, coded, and de-identified. Codebooks, methodology documentation, sample characteristics, analytic strategies, and sample weighting strategies are generally made available by the data providers. Additionally, publicly-available data that is pre-existing and de-identified generally falls under institutional review board (IRB) exemption, thus speeding up the review process.

Nationally representative sampling is another strength of many publicly-available data sets. Sample weighting allows data sets with oversampled subsets to become representative of the larger population. In addition, sample sizes are much larger than those typically feasible for an individual research team to collect. Large samples sizes enable the use of more advanced statistical methods such as multilevel modeling, structural equation modeling, and propensity score matching, all of which improve our understanding of the interrelatedness of concepts and our ability to make causal inferences (Stuart, 2010) about topics that cannot be examined with randomized controlled trials. These advanced methods provide stronger support than simple correlations for targeted intervention research and clinical practice change.

Cost, efficiency, and ethical considerations are interrelated concepts that must be addressed when discussing big data. By their nature, these data are already collected and many are available as no-cost or low-cost alternatives to researchers during funding gaps or as preliminary evidence to shore up grant applications. Perhaps more importantly, the efficient use of such data is an important consideration, given the limited resources available for research. This efficiency of use is further supported by the ethical duty of researchers to participants, who have generously given their time, information and and sometimes biological samples for the express purpose of addressing important research questions. Failure to use these data efficiently may expose others to risks, even if minimal, that are ethically unacceptable if the research could have been carried out from pre-existing data.

\section{Challenges for Researchers and Clinicians}

While the benefits of publicly-available big data are substantial, a few challenges and limitations also exist. Because of the nature of big data, specialized statistical expertise is usually required in order to ensure that the appropriate methods and interpretations are used. Sample weights provided serve to control statistical bias associated with measuring a small subset of a larger population by adjusting the sample pool to more closely match the larger population on a number of characteristics (Solon, Haider, \& Wooldridge, 2015). Good weighting strategies improve the generalizability of observational data, but these require some expertise to handle appropriately. Null hypothesis tests of significance, such as ANOVA and $t$-tests, rely in part on sample size to determine statistical power (Lomax \& 
Hahs-Vaughn, 2012). In very large samples with thousands or even hundreds of thousands of participants, these types of statistics may report statistical significance where no clinical significance exists because the size of the sample simply overpowers the tests. To counter this, the inclusion of effect size determinants both in interpretation of the findings and in reporting is needed. In addition, care must be taken to insure that the data meet the assumptions of the statistical methods that are used. Many data sets are provided in multiple statistical package formats, however some are provided only in one format or are suggested for use with a particular software package. For these reasons, collaboration with a statistician or methodologist who is familiar both with big data sets and sample weighting is highly recommended.

Measurement may also pose a challenge to researchers looking to leverage big data. Specifically, as study designs are predetermined by others, the "ideal" measure of a construct or phenomenon may not have been used to generate the data. Researchers and clinicians attempting to answer clinical questions may have to familiarize themselves with unfamiliar tools and measures or they may need to consider alternative data sets, all of which may require additional researcher time and effort. While some big data collection teams welcome input from "outside" researchers about additional measures and collaborative data collection for future data collection periods, others do not allow researchers to add measures or collect additional data, limiting the type of questions that can be asked.

Finally, with few exceptions, publicly-available data are observational, meaning they are often inappropriate for answering research questions about interventions. While statistical methods such as propensity score matching may improve the ability of researchers to make causal inference (Stuart, 2010), the observational nature of these data may simply offer insight into areas for future research.

\section{Publicly-Available Data with Children's Mental Health Measures}

An ever-increasing number of data sets are available that contain measures, survey questions, and assessments on children's growth, development, physical health, and behavioral and mental health. Examples of how big data has already made a big difference for children can be seen in use in pediatric practices around the nation. Pediatric growth charts were originally established and are regularly updated using data from the National Health and Nutrition Examination Survey (NHANES) which completes comprehensive biological, health, and survey examinations on 5,000 people every year, a subset of whom are children (Centers for Disease Control and Prevention, 2013). The NHANES data has also provided prevalence figures for pediatric diseases and national recommendations on nutrition, and has driven policy changes that led to a national reduction in blood lead content in children through the elimination of lead in gasoline (Centers for Disease Control and Prevention, 2013).

When it comes to pediatric mental health, however, the use of big data has lagged somewhat behind. NHANES includes a number of measures of pediatric mental health, but PubMed searches in June 2014 with the terms "NHANES" and "children" returned fewer than 300 
results when paired with "mental health", yet over 3,000 results when paired with the terms (obesity or weight). Table 1 illustrates the key factors of four publicly-available data sources that include mental health and behavioral measures for children. Of these example studies, two are ongoing, longitudinal studies and two use cross-sectional samples in multiple data waves, each with unique participants. All of these big data sets contain demographic information and at least some measure of mental health or behavior.

For example, the Fragile Families and Child Wellbeing Study is a joint venture between researchers at Princeton University and Columbia University originally constructed to enable study of the influence of child welfare and paternal support policies on child wellbeing (Center for Research on Child Wellbeing, 2015). The currently available public data includes measures of cognition and survey data about school performance, social behaviors, and mental health on 4,898 children followed longitudinally from birth through age 9 , with a retention rate above $70 \%$. This rich body of data is linked to demographic information, social policy information, parental health and incarceration data, and neighborhood characteristics. Additional data including data extracted from medical records about the child's birth, selected genetic markers, school characteristics, and geographical codes are available for restricted use with IRB approval and a nominal fee. Ongoing research by the study group is currently collecting data about the children at age 15 and will reportedly include genetic and biological measures as well as updated survey data. Researchers have previously used these data to address research questions related to the effects on children of parental incarceration, nonresident fathers, racial disparities, and exposure to violence (Center for Research on Child Wellbeing, 2015).

\section{Conclusion}

Big data sets offer a wealth of information that has formed the basis for our understanding of and practice around child health for decades. Some researchers have already begun addressing questions about child mental health from these data, yet much of the potential power of these data sets remains to be tapped. With the growing prevalence of complex pediatric mental health problems and the serious effects that these problems have on quality of life throughout the lifespan, a host of clinical and research questions have sprung up which demand prompt answers. Big data, and publicly-available data in particular, offer researchers and clinicians a pathway to addressing these questions about child mental health rapidly, cheaply, ethically, and in a way that supports wide generalizability. More information about big data and health care is available through the BD2K website (https:// datascience.nih.gov/bd2k, National Institutes of Health, 2015) and at the Data.gov website (data.gov, 2015).

\section{Acknowledgments}

Funding Acknowledgement: The author was supported by a Ruth L. Kirschstein National Research Service Award (NRSA) Institutional Research Training Grant (T32NR014225; Arcoleo, PI) from the National Institute of Nursing Research, National Institutes of Health in affiliation with The Ohio State University. 


\section{References}

Center for Research on Child Wellbeing. Fragile Families and Child Wellbeing Study. 2015. Retrieved from http://www.fragilefamilies.princeton.edu/

Centers for Disease Control and Prevention. About the National Health and Nutrition Examination Survey. 2013. Retrieved February 11, 2014, from http://www.cdc.gov/nchs/nhanes/ about_nhanes.htm\#data

Cox, M.; Ellsworth, D. Proceedings of the 8th Conference on Visualization '97. Los Alamitos, CA, USA: IEEE Computer Society Press; 1997. Application-controlled Demand Paging for Out-of-core Visualization; p. 235-ff.Retrieved from http://dl.acm.org/citation.cfm?id=266989.267068

data.gov. Health. 2015. Retrieved from http://www.data.gov/health/

Lomax, RG.; Hahs-Vaughn, DL. An Introduction to Statistical Concepts. 3. New York: Routledge; 2012.

National Institutes of Health. Big Data to Knowledge (BD2K). 2015. Retrieved from https:// datascience.nih.gov/bd2k

Solon G, Haider SJ, Wooldridge JM. What are we weighting for? Journal of Human Resources. 2015; 50(2):301-316. http://doi.org/10.3368/jhr.50.2.301.

Stuart EA. Matching methods for causal inference: A review and a look forward. Statistical Science : A Review Journal of the Institute of Mathematical Statistics. 2010; 25(1):1-21. http://doi.org/ 10.1214/09-STS313. [PubMed: 20871802] 


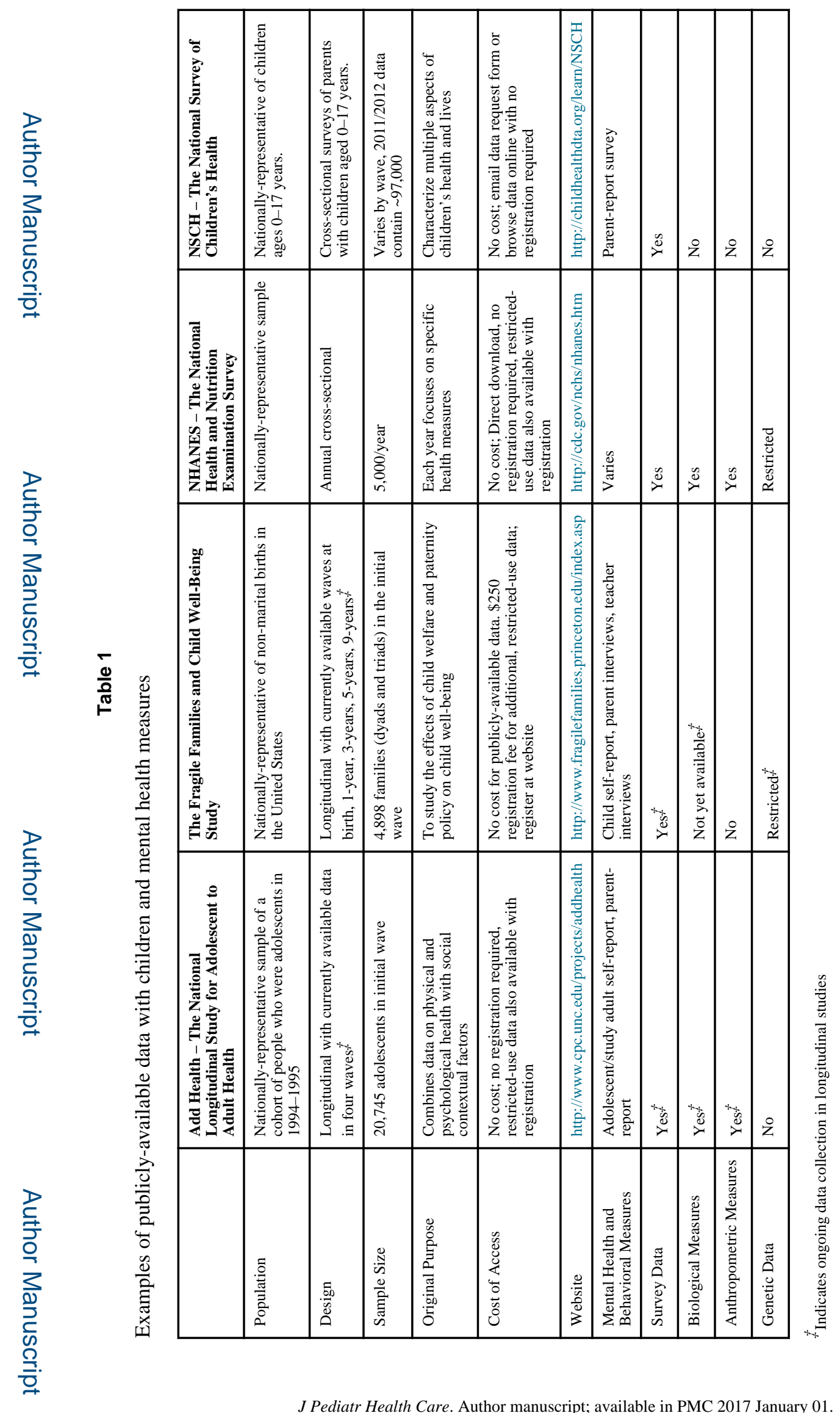

